\title{
A NEW APPROACH TO THE CREATION AND PROPAGATION OF EXPONENTIAL MOMENTS IN THE BOLTZMANN EQUATION
}

\author{
RICARDO ALONSO, JOSÉ A. CAÑIZO, IRENE M. GAMBA, AND CLÉMENT MOUHOT
}

\begin{abstract}
We study the creation and propagation of exponential moments of solutions to the spatially homogeneous $d$-dimensional Boltzmann equation. In particular, when the collision kernel is of the form $\left|v-v_{*}\right|^{\beta} b(\cos (\theta))$ for $\beta \in(0,2]$ with $\cos (\theta)=\left|v-v_{*}\right|^{-1}\left(v-v_{*}\right) \cdot \sigma$ and $\sigma \in \mathbb{S}^{d-1}$, and assuming the classical cut-off condition $b(\cos (\theta))$ integrable in $\mathbb{S}^{d-1}$, we prove that there exists $a>0$ such that moments with weight $\exp \left(a \min \{t, 1\}|v|^{\beta}\right)$ are finite for $t>0$, where $a$ only depends on the collision kernel and the initial mass and energy. We propose a novel method of proof based on a single differential inequality for the exponential moment with time-dependent coefficients.
\end{abstract}

Mathematics Subject Classification (2000): 26D10, 35A23, 76P05, 82C40, $82 \mathrm{D} 10$

Keywords: Boltzmann equation; polynomial moments; exponential moments; Povzner's estimates; differential inequality.

\section{Contents}

1. Introduction

2. Refresher on the sharp Povzner Lemma

3. Proof of the main theorems

Appendix A. Some technical tools on moments

References

\section{INTRODUCTION}

We consider the spatially homogeneous Boltzmann equation in dimension $d \geq 2$ with initial condition $f_{0} \geq 0$, given by

$$
\partial_{t} f=Q(f, f), \quad f(t, \cdot)=f_{0}
$$

where $f=f(t, v) \geq 0$ is a non-negative function depending on time $t \geq 0$ and velocity $v \in \mathbb{R}^{d}$, with $d \geq 2$. We will assume throughout this paper that $f_{0}$ has

RA acknowledges the support from the NSF grant DMS-0439872, IPAM and CAAM. JAC was supported by the project MTM2011-27739-C04 from DGI-MICINN (Spain) and 2009-SGR-345 from AGAUR-Generalitat de Catalunya. IMG has been partially funded by NSF grant DMS1109625 and by DMS FRG-0757450. CM \& JAC acknowledge the support from the ERC grant MATKIT. Support from the Institute from Computational Engineering and Sciences at the University of Texas at Austin is also gratefully acknowledged. 
finite mass and energy, i.e.,

$$
\left\|f_{0}\right\|_{L^{1}\left(1+|v|^{2}\right)}:=\int_{\mathbb{R}^{d}}\left(1+|v|^{2}\right) f_{0}(v) \mathrm{d} v<+\infty .
$$

For $p \in[1,+\infty]$, we denote by $L^{p}$ the Lebesgue spaces of $p$-integrable real functions on $\mathbb{R}^{d}$, and the notation $L^{p}\left(w(v) \mathrm{d} v\right.$ ) (or simply $L^{p}(w(v))$ ) denotes the $L^{p}$ space with weight $w(v)$. The collision operator $Q(f, f)$ is given by

$$
Q(f, f)(v):=\int_{\mathbb{R}^{d} \times \mathbb{S}^{d-1}} B\left(\left|v-v_{*}\right|, \cos \theta\right)\left(f_{*}^{\prime} f^{\prime}-f_{*} f\right) \mathrm{d} v_{*} \mathrm{~d} \sigma,
$$

representing the total rate of binary interactions due to particles taking the direction of $v$ due to collisions, minus those that were knocked out from the $v$ direction. We follow the usual notation $f \equiv f(v), f_{*} \equiv f\left(v_{*}\right), f^{\prime} \equiv f\left(v^{\prime}\right), f_{*}^{\prime} \equiv f\left(v_{*}^{\prime}\right)$. The vectors $v^{\prime}, v_{*}^{\prime}$, which denote the velocities after an elastic collision of particles with velocities $v, v_{*}$, are given by

$$
v^{\prime}:=\frac{v+v_{*}}{2}+\frac{\left|v-v_{*}\right|}{2} \sigma, \quad v_{*}^{\prime}:=\frac{v+v_{*}}{2}-\frac{\left|v-v_{*}\right|}{2} \sigma .
$$

The variable $\theta$ denotes the angle between $v-v_{*}$ and $\sigma$, where $\sigma$ is the unit vector in the direction of the postcollisional relative velocity. On the collision kernel $B$ we assume that for some $\beta \in(0,2]$

$$
B\left(\left|v-v_{*}\right|, \cos \theta\right)=\left|v-v_{*}\right|^{\beta} b(\cos \theta),
$$

with the following cut-off assumption:

$$
b \in L^{1}\left([-1,1],\left(1-z^{2}\right)^{\frac{d-3}{2}} \mathrm{~d} z\right) .
$$

If we define $\tilde{b}(\sigma):=b\left(e_{1} \cdot \sigma\right)$, with $e_{1} \in \mathbb{S}^{d-1}$ any fixed vector, then (3) is equivalent to $\tilde{b} \in L^{1}\left(\mathbb{S}^{d-1}\right)$, which can be easily seen by a spherical change of coordinates.

Throughout the paper $f$ always represents a solution to equation (11) on $[0,+\infty)$ (in the sense of, e.g., [10]) and we always write, for $p \geq 0$ (not necessarily an integer),

$$
m_{p}=m_{p}(t):=\int_{\mathbb{R}^{d}} f(t, v)|v|^{p} \mathrm{~d} v
$$

Main results. It is known that moments of order $p>2$ and exponential moments ( $L^{1}$-exponentially weighted estimates) with weight up to $\exp \left(a|v|^{2}\right)$ for some $a>0$ are propagated by equation (10) [5, 12, 2, 3, 6]; that is, they are finite for all times $t>0$ if they are initially finite, however with a deterioration of the constant $a$. In 12. it was proved that in fact equation (10) with $\beta>0$ instantaneously creates all moments of orders $p>2$, which then remain finite for all times $t>0$. Here the assumption that $\beta>0$ is necessary, since the result is not true for Maxwell molecules for instance 77. Moreover, moments with exponential weight up to $\exp \left(a|v|^{\beta / 2}\right)$ for some constant $a>0$ were also shown to be instantaneously created in 9, 11. In all these proofs it was crucial to assume that the angular function $b$ is in $L^{q}([-1,1],(1-$ $\left.z^{2}\right)^{\frac{d-3}{2}} \mathrm{~d} z$ ) for $q>1$ as done in [4, 6, 11. We also refer to the recent work [8] for moment production estimates in the so-called non-cutoff case, in which proofs are based on the optimization of the traditional inductive argument [2, 3, 6, 9, 11].

We have several noticeable contributions in this paper. Indeed, we can extend the existing propagation and creation of $L^{1}$-exponentially weighted estimates to include 
the classical cut-off assumption $b \in L^{1}\left([-1,1],\left(1-z^{2}\right)^{\frac{d-3}{2}} \mathrm{~d} z\right)$ without using the iterative methods developed in [4, 6, 1]), and also we slightly relax the assumptions on the initial data by requiring only finite mass and energy, and not necessarily finite entropy as in previous works on creation of moments [12]. In addition, we improve the weights for the creation of $L^{1}$-exponentially weighted moments, with a weight up to $\exp \left(a|v|^{\beta}\right)$ (hence removing the $1 / 2$ factor which was present in [9, 11]) for solutions with finite mass and energy, assuming only an integrability condition on $b$. More specifically, Theorem 1 gives an explicit rate of appearance of exponential moments by showing that the coefficient multiplying $|v|^{\beta}$ in the exponential weight can be taken linearly growing in time.

The most important point is that we introduce a new method of proof that not only does not need iterative arguments but also allows for all these improvements. This approach is also used in Theorem 2 for the propagation of exponential moments, and extends these results to classical cut-off assumptions on the angular cross section $b$.

Theorem 1 (Creation of exponential moments). Let $f$ be an energy-conserving solution to the homogeneous Boltzmann equation (1) on $[0,+\infty)$ with initial data $f^{0} \in L^{1}\left(1+|v|^{2}\right)$, and assume (2) and (3) with $\beta \in(0,2]$. Then there are some constants $C, a>0$ (which depend only on $b, \beta$ and the initial mass and energy) such that

$$
\int_{\mathbb{R}^{d}} f(t, v) \exp \left(a \min \{t, 1\}|v|^{\beta}\right) \mathrm{d} v \leq C \quad \text { for } t \geq 0 .
$$

We remark that the existence and uniqueness of energy-conserving solutions with initial data $f^{0} \in L^{1}\left(1+|v|^{2}\right)$ was proved in [10].

As mentioned above, our approach also provides a new proof of the property of propagation of exponential moments 6 , 4]. This is stated in the following theorem:

Theorem 2 (Propagation of exponential moments). Let $f$ be an energy-conserving solution to the homogeneous Boltzmann equation (11) on $[0,+\infty)$ with initial data $f^{0} \in L^{1}\left(1+|v|^{2}\right)$, and assume (2) and (3) with $\beta \in(0,2]$. Assume moreover that the initial data satisfies for some $s \in[\beta, 2]$

$$
\int_{\mathbb{R}^{d}} f_{0}(v) \exp \left(a_{0}|v|^{s}\right) \mathrm{d} v \leq C_{0} .
$$

Then there are some constants $C, a>0$ (which depend only on $b, \beta$ and the initial mass, energy and $a_{0}, C_{0}$ in (5) ) such that

$$
\int_{\mathbb{R}^{d}} f(t, v) \exp \left(a|v|^{s}\right) \mathrm{d} v \leq C \quad \text { for } t \geq 0 .
$$

We give in Section 3 a novel argument for proving these results which is based on a differential inequality for the exponential moment itself, and the exploitation of a discrete convolution-type estimate for the exponential moment of the gain part of the collision operator. This avoids the intricate combination of induction and maximum principle arguments in the previous proofs of propagation [6, 4] and appearance [9, 11] of exponential moments. It also clarifies the structure underlying these induction arguments. The starting point of both these previous works and our approach is the creation and propagation of polynomial moments in [5, 12] 
and the Povzner inequalities proved in 4, 1. We include a short appendix which gathers some of the classical technical results used along the proofs.

\section{Refresher on the Sharp Povzner Lemma}

The following lemma reflects the angular averaging property of the spherical integral acting on positive convex test functions evaluated at the postcollisional velocities. These estimates are crucial to be able to control in a sharp form the moments of the gain operator by estimates for lower bounds of the loss operator. They were originally introduced in [4, Corollary 1] and further developed in [6, Lemma 3 and 4] and more recently in [1, Lemma 2.6]. We summarize these results as follows:

Lemma 3 (Sharp Povzner (angular averaging) Lemma). Assume that $b:(-1,1) \rightarrow$ $[0, \infty)$ satisfies (3), and impose without loss of generality the following normalization condition

$$
\int_{-1}^{1} b(z)\left(1-z^{2}\right)^{\frac{d-3}{2}} \mathrm{~d} z=\frac{1}{\left|\mathbb{S}^{d-2}\right|}
$$

where $\left|\mathbb{S}^{d-2}\right|$ is the area of the $(d-2)$-dimensional unit sphere. Then for $p \geq 1$ it holds that

$$
\int_{\mathbb{S}^{d-1}}\left(\left|v^{\prime}\right|^{2 p}+\left|v_{*}^{\prime}\right|^{2 p}\right) b(\cos \theta) \mathrm{d} \sigma \leq \gamma_{p}\left(|v|^{2}+\left|v_{*}\right|^{2}\right)^{p}
$$

where $\gamma_{p}>0$ are constants such that $\gamma_{1}=1, p \mapsto \gamma_{p}$ is strictly decreasing and tends to 0 as $p \rightarrow \infty$.

Remark 4 . In the case when the symmetrization $z \mapsto b(z)+b(-z)$ of $b$ is nondecreasing in $[0,1]$, these constants are controlled by

$$
\gamma_{p} \leq \frac{1}{\left|\mathbb{S}^{d-2}\right|} \int_{-1}^{1} b(z)\left(\frac{1+z}{2}\right)^{p}\left(1-z^{2}\right)^{\frac{d-3}{2}} \mathrm{~d} z .
$$

Remark 5. In addition, when $b \in L^{q}\left([-1,1],\left(1-z^{2}\right)^{(d-3) / 2} d z\right)$ with $q>1$, the decay of $\gamma_{p}$ can be estimated and shown to be polynomial: there exists a constant $C>0$ such that

$$
\gamma_{p} \leq \min \left\{1, \frac{C}{p^{1 / q^{\prime}}}\right\} \quad(p>1)
$$

with $q^{\prime}$ the Hölder dual of $q$ (i.e., $1 / q+1 / q^{\prime}=1$ ). Furthermore, in the case $q=+\infty$, that is, for $b$ bounded, it holds that

$$
\gamma_{p} \leq \min \left\{1, \frac{16 \pi b^{*}}{p+1}\right\} \quad(p>1)
$$

with $b^{*}:=\max _{-1 \leq z \leq 1} b(z)$.

Let us now state the key a priori estimate on the polynomial moments, which shall be used in the sequel. For later reference, we define the following quantity for any $s, p>0$ :

$$
S_{s, p}=S_{s, p}(t):=\sum_{k=1}^{k_{p}}\left(\begin{array}{l}
p \\
k
\end{array}\right)\left(m_{s k+\beta} m_{s(p-k)}+m_{s k} m_{s(p-k)+\beta}\right),
$$

with $k_{p}$ the integer part of $(p+1) / 2$. 
Lemma 6 (A priori estimate on the polynomial moments). For $s \in(0,2]$ and $p_{0}>2 / s$, the following a priori inequality is true whenever all the terms make sense:

$$
\frac{\mathrm{d}}{\mathrm{d} t} m_{s p} \leq 2 \gamma_{s p / 2} S_{s, p}-K_{1} m_{s p+\beta}+K_{2} m_{s p} \quad \text { for } t \geq 0, p \geq p_{0}>\frac{2}{s},
$$

with $S_{s, p}$ given by (9) and constants

$$
K_{1}:=2\left(1-\gamma_{s p_{0} / 2}\right) C_{\beta} m_{0} \quad \text { and } \quad K_{2}=2 m_{\beta}
$$

with $C_{\beta}:=\min \left\{1,2^{1-\beta}\right\}$.

Alternatively in the case $\beta \in(0,1]$, it is possible to get rid of the second constant, and obtain

$$
K_{1}:=2\left(1-\gamma_{s p_{0} / 2}\right) \bar{C}_{\beta} m_{0} \quad \text { and } \quad K_{2}=0
$$

for some constant $\bar{C}_{\beta}$ depending on $\beta$ and the initial data.

In both cases, the constant $\gamma_{s p_{0} / 2}$ depends on the integrability of the angular function $b$ and on $p_{0}>2 / s$.

Proof. Using Lemma 3 one obtains for any $p \geq 2 / s$ :

$$
\begin{aligned}
& \frac{\mathrm{d}}{\mathrm{d} t} m_{s p} \\
& \begin{aligned}
\leq \gamma_{s p / 2} \int_{\mathbb{R}^{d} \times \mathbb{R}^{d}} f f_{*}\left(\left(|v|^{2}\right.\right. & \left.\left.+\left|v_{*}\right|^{2}\right)^{\frac{s p}{2}}-|v|^{s p}-\left|v_{*}\right|^{s p}\right)\left|v-v_{*}\right|^{\beta} \mathrm{d} v \mathrm{~d} v_{*} \\
& \quad-2\left(1-\gamma_{s p / 2}\right) \int_{\mathbb{R}^{d} \times \mathbb{R}^{d}} f f_{*}|v|^{s p}\left|v-v_{*}\right|^{\beta} \mathrm{d} v \mathrm{~d} v_{*} .
\end{aligned}
\end{aligned}
$$

In order to estimate the right hand side of (13) we first focus on an upper bound for its positive term. Since $0<s / 2 \leq 1$, then

$$
\left(|v|^{2}+\left|v_{*}\right|^{2}\right)^{\frac{s p}{2}} \leq\left(|v|^{s}+\left|v_{*}\right|^{s}\right)^{p}
$$

Hence, using Lemma 11 in the Appendix (a classical result taken from [4, Lemma 2]) and the estimate $\left|v-v_{*}\right|^{\beta} \leq 2|v|^{\beta}+2\left|v_{*}\right|^{\beta}$ we obtain that, for any $p \geq 1$, the first integral in (13) is controlled by

$$
\begin{aligned}
\gamma_{s p / 2} \int_{\mathbb{R}^{d} \times \mathbb{R}^{d}} f f_{*}\left(\left(|v|^{2}+\left|v_{*}\right|^{2}\right)^{\frac{s p}{2}}-|v|^{s p}-\left|v_{*}\right|^{s p}\right)\left|v-v_{*}\right|^{\beta} \mathrm{d} v & \mathrm{~d} v_{*} \\
& \leq 2 \gamma_{s p / 2} S_{s, p} .
\end{aligned}
$$

The estimate of the negative term in (13) requires a control from below. When $\beta \in(0,1]$ it follows from Lemma 12 in the Appendix (taken from [6. Lemma 2]) that the lower bound for the negative term in (13) satisfies

$$
2\left(1-\gamma_{s p / 2}\right) \int_{\mathbb{R}^{d} \times \mathbb{R}^{d}} f f_{*}|v|^{s p}\left|v-v_{*}\right|^{\beta} \mathrm{d} v \mathrm{~d} v_{*} \geq 2 \bar{C}_{\beta}\left(1-\gamma_{s p / 2}\right) m_{0} m_{s p+\beta}
$$

for some constant $\bar{C}_{\beta}$ related to $\beta$ and the initial data. So estimate (10) follows with $K_{1}$ and $K_{2}$ as in (12).

In the general case $\beta \in(0,2]$, the previous argument does not necessarily follow, yet it is still possible to obtain an easier lower bound that still allows for the control of moments and their summability. We use the fact that $\left|v-v_{*}\right|^{\beta} \geq 2^{1-\beta}|v|^{\beta}-\left|v_{*}\right|^{\beta}$ 
(which can be obtained from the triangle inequality and the inequality $(x+y)^{\beta} \leq$ $C_{\beta}^{-1}\left(x^{\beta}+y^{\beta}\right)$ for $x, y \geq 0$.) This gives a lower bound for the negative term in (13):

$$
\begin{aligned}
2\left(1-\gamma_{s p / 2}\right) \int_{\mathbb{R}^{d} \times \mathbb{R}^{d}} f f_{*}|v|^{s p}\left|v-v_{*}\right|^{\beta} \mathrm{d} v \mathrm{~d} v_{*} \\
\geq 2\left(1-\gamma_{s p / 2}\right) C_{\beta} m_{0} m_{s p+\beta}-2 m_{\beta} m_{s p} .
\end{aligned}
$$

Since $\gamma_{s p}$ decreases as $p \rightarrow \infty$, it follows that $2\left(1-\gamma_{s p / 2}\right) C_{\beta} m_{0} \geq K_{1}$ for any $p \geq p_{0}$. Hence, estimate (10) follows with $K_{1}$ and $K_{2}$ as in (11).

Remark 7. We note that neither in the work [6] nor in here the finiteness of the entropy is required, however it was needed in the earlier work 12 in order to obtain lower bounds for the negative term in (13). If the solution has a finite entropy, then these lower bounds may be obtained by the same technique as in [12]. Observe however that the constant $\bar{C}_{\beta}$ in the case $\beta \in(0,1]$ with $K_{2}=0$ depends on the initial data in a non-trivial way, through the positive constant $C>0$ such that

$$
\int_{\mathbb{R}^{d}} f_{0}\left(v_{*}\right)\left|v-v_{*}\right|^{\beta} \mathrm{d} v_{*} \geq C\left(1+|v|^{\beta}\right)
$$

which cannot be expressed simply in terms of the mass and energy of $f_{0}$. Nevertheless the general argument (involving $K_{2}>0$ ) does provide constants only depending on the initial data through its mass and momentum.

Next, we recall and prove a very similar result to that in [12, Theorem 4.2]. The main difference is that finiteness of the entropy of the initial condition is not required here.

Lemma 8 (Creation and propagation of polynomial moments). Assume (2) and (33) with $0<\beta \leq 2$. Set $s \in(0,2]$, and let $f$ be an energy-conserving solution to the homogeneous Boltzmann equation (1) with initial data $f_{0} \in L^{1}\left(1+|v|^{2}\right)$. For every $p>0$ there exists a constant $C_{s p} \geq 0$ depending only on $p, s, b$ and the initial mass and energy, such that

$$
m_{s p}(t) \leq C_{s p} \max \left\{1, t^{-s p / \beta}\right\} \quad \text { for } t>0 .
$$

If $m_{s p}(0)$ is finite, then the control can be improved to simply

$$
m_{s p}(t) \leq C_{s p} \quad \text { for } t \geq 0
$$

for some constant $C_{s p}$ depending only on $p, s, b$, the initial mass and energy, and $m_{p}(0)$.

Proof. Following a common procedure (see [10, 12]), the estimates can be carried first on a truncated solution (for which all moments are finite and our calculations are rigorously justified), and then proved for the solution to the full problem by relaxing the truncation parameter.

Let us prove (17): observe that by Hölder's inequality

$$
S_{s, p} \leq C m_{\beta} m_{s p} \quad \text { and } \quad m_{s p+\beta} \geq K m_{s p}^{1+\beta /(s p)}
$$

for some constants $C, K>0$ depending only on $s, p$, the initial mass and energy. Since $\beta \leq 2$, we have $1 \leq 2 / \beta$ and therefore $m_{\beta}$ is controlled by the mass and energy. We deduce that $m_{s p}$ satisfies the differential inequality

$$
\frac{\mathrm{d}}{\mathrm{d} t} m_{s p} \leq C^{\prime} m_{s p}-K m_{s p}^{1+\beta /(s p)}
$$


for some other constant $C^{\prime}>0$ depending only on $s, p$, the initial mass and energy. This readily implies the bound (17) by computing an upper solution to this differential inequality, and the bound (18) by a maximum principle argument.

Remark 9. Observe that the polynomial bound $O\left(t^{-s p / \beta}\right)$ on the appearance of $m_{p}$ is not optimal, as can be seen from [10, Theorem 1.1]. However our rate of appearance of exponential moments can be seen to be optimal by inspection of the simpler equation $\partial_{t} f=-C\left(1+|v|^{\beta}\right) f$ which provides subsolutions to the Boltzmann equation.

\section{Proof of the MAIN TheOREMS}

In this section we give a proof of Theorems 1 and 2 valid for any integrable crosssection $b$. We first carry out the estimates on a finite sum of polynomial moments, and then pass to the limit.

Our goal is to estimate the quantity

$$
E_{s}(t, z):=\int_{\mathbb{R}^{d}} f(t, v) \exp \left(z|v|^{s}\right) \mathrm{d} v=\sum_{p=0}^{\infty} m_{s p}(t) \frac{z^{p}}{p !}
$$

where $s=\beta$ and $z=a t$ for Theorem 1 and $s \in(0,2]$ and $z=a$ for Theorem 2 , for some $a>0$. For use below let us define the truncated sum as

$$
E_{s}^{n}(t, z):=\sum_{p=0}^{n} m_{s p}(t) \frac{z^{p}}{p !}
$$

for $n \in \mathbb{N}, z \geq 0$, and $t \geq 0$. We also define

$$
I_{s, \beta}^{n}(t, z):=\sum_{p=0}^{n} m_{s p+\beta}(t) \frac{z^{p}}{p !} .
$$

Let us first prove the key lemma, which identifies the discrete convolution structure. This result gives a control for finite sums of the moments associated to the gain operator. It is uniform in $\beta \in(0,2]$ :

Lemma 10. Assume $0<\beta \leq s \leq 2$. For any $p_{0} \geq 2 / s$, we have the following functional inequality

$$
\sum_{p=p_{0}}^{n} \frac{z^{p}}{p !} S_{s, p}(t) \leq 2 E_{s}^{n}(t, z) I_{s, \beta}^{n}(t, z)
$$

where $S_{s, p}$ was defined in (9).

Proof. Let us recall the definition of $S_{s, p}$ from (9):

$$
S_{s, p}:=\sum_{k=1}^{k_{p}}\left(\begin{array}{l}
p \\
k
\end{array}\right)\left(m_{s k+\beta} m_{s(p-k)}+m_{s k} m_{s(p-k)+\beta}\right),
$$


where $k_{p}$ is the integer part of $(p+1) / 2$. The first part of the sum in the left hand side of (20) can be bounded as:

$$
\begin{aligned}
\sum_{p=p_{0}}^{n} \frac{z^{p}}{p !} \sum_{k=1}^{k_{p}} & \left(\begin{array}{l}
p \\
k
\end{array}\right) m_{s k+\beta} m_{s(p-k)}=\sum_{p=p_{0}}^{n} \sum_{k=1}^{k_{p}} m_{s k+\beta} \frac{z^{k}}{k !} m_{s(p-k)} \frac{z^{p-k}}{(p-k) !} \\
\leq & \sum_{k=1}^{n} m_{s k+\beta} \frac{z^{k}}{k !} \sum_{p=\max \left\{p_{0}, 2 k-1\right\}}^{n} m_{s(p-k)} \frac{z^{p-k}}{(p-k) !} \leq I_{s, \beta}^{n}(t, z) E_{s}^{n}(t, z) .
\end{aligned}
$$

We carry out a similar estimate for the other part:

$$
\begin{aligned}
\sum_{p=p_{0}}^{n} \frac{z^{p}}{p !} \sum_{k=1}^{k_{p}} & \left(\begin{array}{l}
p \\
k
\end{array}\right) m_{s k} m_{s(p-k)+\beta}=\sum_{p=p_{0}}^{n} \sum_{k=1}^{k_{p}} m_{s k} \frac{z^{k}}{k !} m_{s(p-k)+\beta} \frac{z^{p-k}}{(p-k) !} \\
& \leq \sum_{k=1}^{n} m_{s k} \frac{z^{k}}{k !} \sum_{p=\max \left\{p_{0}, 2 k-1\right\}}^{n} m_{s(p-k)+\beta} \frac{z^{p-k}}{(p-k) !} \leq E_{s}^{n}(t, z) I_{s, \beta}^{n}(t, z)
\end{aligned}
$$

which concludes the proof.

We now can prove both Theorem 1 and Theorem 2, We write the proof first for the case $\beta \in(0,1]$ with the choice of constants (12) in (10) (hence with $K_{2}=0$ ). Later we show the corresponding estimates for the full range $\beta \in(0,2]$ using the choice of constants (11) in (10).

Proof of Theorem 11. First we notice that it is enough to prove the following (under the same assumptions): there are some constants $T, C, a>0$ (which depend only on $b$ and the initial mass and energy) such that

$$
\int_{\mathbb{R}^{d}} f(t, v) \exp \left(a t|v|^{\beta}\right) \mathrm{d} v \leq C \quad \text { for } t \in[0, T] .
$$

Indeed, since the assumptions of lower and upper bounds on the mass and energy are satisfied uniformly in time along the flow, for $t \geq T$ it is then possible to apply (21) starting at time $(t-T)$.

Hence, we aim at proving the estimate (21). We set $s=\beta$. Consider $a>0$ to be fixed later, $n \in \mathbb{N}$ and define $T>0$ as

$$
T:=\min \left\{1 ; \sup \left\{t>0 \text { s.t. } E_{\beta}^{n}(t, a t)<4 m_{0}\right\}\right\} .
$$

The definition is consistent since $E_{\beta}^{n}(0,0)=m_{0}$ and the Lemma 8 ensures that $T>0$ for each given $n$. The bound of 1 is not essential, and is included just to ensure that $T$ is finite. We note that a priori such $T$ depends on the index $n$ in the sum $E_{\beta}^{n}$. However, we will show that $T$ has a lower bound that depends only on $b, \beta$ and the initial mass and energy, thus proving the theorem. Unless otherwise noted, all equations below which depend on time are valid for $t \in[0, T]$.

Choose an integer $p_{0}>2 / \beta$, to be fixed later. Starting from Lemma 6 (inequality (10), we have

$$
\frac{\mathrm{d}}{\mathrm{d} t} m_{\beta p} \leq 2 \gamma_{\beta p / 2} S_{\beta, p}-K_{1} m_{\beta(p+1)} \quad \text { for } t \geq 0, p \geq p_{0},
$$

with $S_{\beta, p}$ given by (9) and $K_{1}$ defined in (12), independent of $p$ with $p \geq p_{0}$ as soon as $p_{0}$ is strictly bounded away from $2 / \beta$. 
In addition, from Lemma 8 (inequality (17)) we know that there exists a constant $C_{p_{0}}>0$ (depending on $p_{0}$ ) such that

$$
\sum_{p=0}^{p_{0}} m_{\beta p}(t) t^{p} \leq C_{p_{0}} \quad \text { for all } t \in[0, T]
$$

Taking any $a<1$ and using the product rule,

$$
\begin{aligned}
& \frac{\mathrm{d}}{\mathrm{d} t} \sum_{p=p_{0}}^{n} m_{\beta p} \frac{(a t)^{p}}{p !} \\
& \quad \leq \sum_{p=p_{0}}^{n} \frac{(a t)^{p}}{p !}\left(2 \gamma_{\beta p / 2} S_{\beta, p}-K_{1} m_{\beta(p+1)}\right)+a \sum_{p=p_{0}}^{n} m_{\beta p} \frac{(a t)^{p-1}}{(p-1) !} \\
& \leq 2 \sum_{p=p_{0}}^{n} \frac{(a t)^{p}}{p !} \gamma_{\beta p / 2} S_{\beta, p}+\left(a-K_{1}\right) I_{\beta, \beta}^{n}(t, a t)+\left(K_{1}+a\right) \sum_{p=1}^{p_{0}} m_{\beta p} \frac{(a t)^{p-1}}{(p-1) !} \\
& \quad \leq 2 \sum_{p=p_{0}}^{n} \frac{(a t)^{p}}{p !} \gamma_{\beta p / 2} S_{\beta, p}+\left(a-K_{1}\right) I_{\beta, \beta}^{n}(t, a t)+\frac{1}{t}\left(K_{1}+a\right) C_{p_{0}},
\end{aligned}
$$

where we have used $a<1$ and (23) in the last step. Hence, from Lemma 10 (inequality (20) ) we obtain

$$
\frac{\mathrm{d}}{\mathrm{d} t} \sum_{p=p_{0}}^{n} m_{\beta p} \frac{(a t)^{p}}{p !} \leq I_{\beta, \beta}^{n}(t, a t)\left[4 \gamma_{\beta p_{0} / 2} E_{\beta}^{n}(t, a t)+\left(a-K_{1}\right)\right]+\frac{1}{t}\left(K_{1}+a\right) C_{p_{0}} .
$$

Next, choose $p_{0}$ large enough such that $16 \gamma_{\beta p_{0} / 2} m_{0} \leq(1 / 4) K_{1}$ (or equivalently, by using the definition of $K_{1}$ in (11), $\left.\gamma_{\beta p_{0} / 2}<\left(32+\bar{C}_{\beta}\right)^{-1}\right)$ and restrict further the parameter $a$, so that $a \leq K_{1} / 2$. Then, as $E_{\beta}^{n}(t, a t) \leq 4 m_{0}$ for $t \in[0, T]$, by the definition of $T$ we have

$$
\begin{aligned}
\frac{\mathrm{d}}{\mathrm{d} t} \sum_{p=p_{0}}^{n} m_{\beta p} \frac{(a t)^{p}}{p !} \leq-\frac{1}{4} K_{1} I_{\beta, \beta}^{n}(t, a t) & +\frac{1}{t}\left(K_{1}+a\right) C_{p_{0}} \\
& \leq-\frac{1}{t}\left(\frac{K_{1}}{4 a}\left(E_{\beta}^{n}(t, a t)-m_{0}\right)-\left(K_{1}+a\right) C_{p_{0}}\right)
\end{aligned}
$$

where for the last inequality we have used that

$$
I_{\beta, \beta}^{n}(t, a t) \geq \frac{\left(E_{\beta}^{n}(t, a t)-m_{0}\right)}{a t} .
$$

We make the additional restriction that $a<m_{0} /\left(6 C_{p_{0}}\right)$, which together with $a<$ $K_{1} / 2$ implies that

$$
\frac{K_{1}}{4 a} m_{0}>\left(K_{1}+a\right) C_{p_{0}}
$$

Then, whenever $E_{\beta}^{n}(t, a t) \geq 2 m_{0}$,

$$
\frac{\mathrm{d}}{\mathrm{d} t} \sum_{p=p_{0}}^{n} m_{\beta p} \frac{(a t)^{p}}{p !} \leq 0
$$


for any time $t \in[0, T]$ for which $E_{\beta}^{n}(t, a t) \geq 2 m_{0}$ holds. This is true in particular when $\sum_{p=p_{0}}^{n} m_{\beta p} \frac{(a t)^{p}}{p !} \geq 2 m_{0}$. We deduce that

$$
\sum_{p=p_{0}}^{n} m_{\beta p} \frac{(a t)^{p}}{p !} \leq 2 m_{0} \quad \text { for } t \in[0, T] .
$$

In order to finish the argument we need to bound the initial part of the full sum (from $p=0$ to $p_{0}-1$.) Indeed, we note that from (23),

$$
\sum_{p=0}^{p_{0}-1} m_{\beta p} \frac{(a t)^{p}}{p !} \leq m_{0}+a C_{p_{0}} \quad \text { for } t \in[0, T]
$$

so, recalling that $6 a C_{p_{0}}<m_{0}$ and using (26) and (27)

$$
E_{\beta}^{n}(t, a t)=\sum_{p=0}^{p_{0}-1} m_{\beta p} \frac{(a t)^{p}}{p !}+\sum_{p=p_{0}}^{n} m_{\beta p} \frac{(a t)^{p}}{p !} \leq 3 m_{0}+a C_{p_{0}} \leq \frac{19}{6} m_{0}
$$

for $t \in[0, T]$, uniformly in $n$. Finally, gathering all conditions imposed along the proof on the parameter $a$, we choose

$$
a:=\min \left\{1, \frac{K_{1}}{2}, \frac{m_{0}}{6 C_{p_{0}}}\right\}
$$

independently of $n$, where $K_{1}$ was defined in (12) and $C_{p_{0}}$ in (23). We conclude, from the definition of $T$, that $T=1$ for all $n$. Sending $n \rightarrow \infty$, Theorem 1 follows.

In the general case $\beta \in(0,2]$, since $K_{2}$ in (11) is not zero, equation (22) has an extra term in the right hand side, namely

$$
\frac{\mathrm{d}}{\mathrm{d} t} m_{\beta p} \leq 2 \gamma_{\beta p / 2} S_{\beta, p}-K_{1} m_{\beta(p+1)}+K_{2} m_{\beta p} \quad \text { for } t \geq 0, p \geq p_{0} .
$$

In this case using again that $E_{\beta}^{n}(t, a t) \leq 4 m_{0}$ on $[0, T]$, (24) is now modified as

$$
\begin{aligned}
\frac{\mathrm{d}}{\mathrm{d} t} \sum_{p=p_{0}}^{n} m_{\beta p} \frac{(a t)^{p}}{p !} & \leq-\frac{1}{4} K_{1} I_{\beta, \beta}^{n}(t, a t)+\frac{1}{t}\left(K_{1}+a\right) C_{p_{0}}+K_{2} E_{\beta}^{n}(t, a t) \\
& \leq-\frac{1}{t}\left(\frac{K_{1}}{4 a}\left(E_{\beta}^{n}(t, a t)-m_{0}\right)-\left(K_{1}+a\right) C_{p_{0}}\right)+4 K_{2} m_{0} .
\end{aligned}
$$

Hence by tuning the constants as before, at any time $t \in[0, T]$ for which $E_{\beta}^{n}(t, a t) \geq$ $2 m_{0}$ we have

$$
\frac{\mathrm{d}}{\mathrm{d} t} \sum_{p=p_{0}}^{n} m_{\beta p} \frac{(a t)^{p}}{p !} \leq K_{3}
$$

with $K_{3}=4 K_{2} m_{0}$. The corresponding to equation (26) is then

$$
\sum_{p=p_{0}}^{n} m_{\beta p} \frac{(a t)^{p}}{p !} \leq 2 m_{0}+K_{3} t \quad t \in[0, T] .
$$

It follows as before that

$$
E_{\beta}^{n}(t, a t) \leq \frac{19}{6} m_{0}+K_{3} t, \quad t \in[0, T],
$$

uniformly in $n$. Then $T \geq m_{0} /\left(2 K_{3}\right)$, where $K_{3}$ is a constant which depends only on $b$, the hard potential exponent $\beta$ and initial mass and energy. In particular for 
the same rate $a$ as in (28) the conclusion follows since both $a$ and $T$ are uniform in the index $n$ and the limit in $n$ can be performed as well.

Proof of Theorem 2, Consider again first the case $\beta \in(0,1]$, and $s \in[\beta, 1]$ as in (15), $a>0$ to be fixed later and $n \in \mathbb{N}$. Define $T>0$ as

$$
T:=\sup \left\{t>0 \text { s.t. } E_{s}^{n}(t, a)<4 m_{0}\right\} .
$$

This definition is consistent since $E_{s}^{n}(0, a) \leq E_{s}(0, a)<4 m_{0}$ for $a$ small enough thanks to the assumption (5) on the initial data, and the Lemma 8 ensures that $T>0$ for each given $n$. We will show that, for $a$ chosen small enough, $T=+\infty$ for any $n$, thus proving the theorem.

Choose an integer $p_{0}>2 / s$, to be fixed later. Starting again from Lemma 6 (inequality (10) with the choice of constants (12)), we have

$$
\frac{\mathrm{d}}{\mathrm{d} t} m_{s p} \leq 2 \gamma_{s p / 2} S_{s, p}-K_{1} m_{s p+\beta} \quad \text { for } t \geq 0, p \geq p_{0},
$$

with $S_{s, p}$ given by (9) and $K_{1}$ given by (12), independent of $p$ with $p \geq 0$. Also, from Lemma 8 (inequality (18)) we know that there exists a constant $C_{s, p_{0}}>0$ (depending on $s, p_{0}$ ) such that

$$
\sum_{p=0}^{p_{0}} m_{s p} \leq C_{s, p_{0}} \quad \text { for all } t \in[0, T]
$$

Taking any $a<\min \left\{1, a_{0}\right\}$, we have

$$
\begin{aligned}
& \frac{\mathrm{d}}{\mathrm{d} t} \sum_{p=p_{0}}^{n} m_{s p} \frac{a^{p}}{p !} \leq \sum_{p=p_{0}}^{n} \frac{a^{p}}{p !}\left(2 \gamma_{s p / 2} S_{s, p}-K_{1} m_{s p+\beta}\right) \\
& \leq 2 \sum_{p=p_{0}}^{n} \frac{a^{p}}{p !} \gamma_{s p / 2} S_{s \cdot p}-K_{1} I_{s, \beta}^{n}(t, a)+K_{1} \sum_{p=0}^{p_{0}-1} m_{s p+\beta} \frac{a^{p}}{p !} \\
& \leq 2 \sum_{p=p_{0}}^{n} \frac{a^{p}}{p !} \gamma_{s p / 2} S_{s, p}-K_{1} I_{s, \beta}^{n}(t, a)+K_{1} C_{s, p_{0}},
\end{aligned}
$$

where we have used $a<1$ and (31) in the last step. Hence, from Lemma 10 (inequality (20) ) we obtain

$$
\frac{\mathrm{d}}{\mathrm{d} t} \sum_{p=p_{0}}^{n} m_{s p} \frac{a^{p}}{p !} \leq I_{s, \beta}^{n}(t, a)\left[4 \gamma_{s p_{0}} E_{s}^{n}(t, a)-K_{1}\right]+K_{1} C_{s, p_{0}}
$$

where, as in the previous proof, we also choose $p_{0}$ such that $16 \gamma_{s p_{0} / 2} m_{0} \leq(1 / 2) K_{1}$. Then, as $E_{s}^{n}(t, a) \leq 4 m_{0}$ for $t \in[0, T]$ by definition of $T$ we have

$$
\frac{\mathrm{d}}{\mathrm{d} t} \sum_{p=p_{0}}^{n} m_{s p} \frac{a^{p}}{p !} \leq-\frac{1}{2} K_{1} I_{s, \beta}^{n}(t, a)+K_{1} C_{s, p_{0}} \leq-\frac{K_{1}}{2 a} E_{s}^{n}(t, a)+K_{1}\left(\frac{m_{0}}{2 a}+e^{a}\right)+K_{1} C_{s, p_{0}}
$$


where for the last inequality we have used that

$$
\begin{aligned}
I_{s, \beta}^{n}(t, a) \geq \int_{|v| \geq 1}\left(\sum_{p=1}^{n}|v|^{s p+\beta} \frac{a^{p}}{p !}\right) f \mathrm{~d} v \geq \int_{|v| \geq 1}\left(\sum_{p=1}^{n}|v|^{s p} \frac{a^{p}}{p !}\right) f \mathrm{~d} v \\
\geq \int_{\mathbb{R}^{d}}\left(\sum_{p=1}^{n}|v|^{s p} \frac{a^{p}}{p !}\right) f \mathrm{~d} v-e^{a} \int_{\mathbb{R}^{d}} f \mathrm{~d} v \geq \frac{\left(E_{s}^{n}(t, a)-m_{0}\right)}{a}-e^{a},
\end{aligned}
$$

so that

$$
\frac{\mathrm{d}}{\mathrm{d} t} \sum_{p=p_{0}}^{n} m_{s p} \frac{a^{p}}{p !} \leq-\frac{K_{1}}{2 a} E_{s}^{n}(t, a)+K_{1}\left(\frac{m_{0}}{2 a}+e^{a}\right)+K_{1} C_{s, p_{0}} .
$$

Next, recalling estimate (19) in the proof of Lemma 8

$$
\frac{\mathrm{d}}{\mathrm{d} t} m_{s p} \leq C^{\prime} m_{s p}
$$

valid for any $p \in \mathbb{N}$ and constant $C^{\prime}$ depending only on $s, p$, initial mass and energy. Summing in $p$, from 0 to $p_{0}-1$, and using estimate (18) we obtain

$$
\frac{\mathrm{d}}{\mathrm{d} t} E_{s}^{n}(t, a) \leq-\frac{K_{1}}{2 a} E_{s}^{n}(t, a)+K_{1}\left(\frac{m_{0}}{2 a}+e^{a}\right)+\left(K_{1}+C^{\prime}\right) C_{s, p_{0}} .
$$

This implies, by a maximum principle argument for ODEs, that the bound

$$
E_{s}^{n}(t, a) \leq m_{0}+2 a\left[\left(1+\frac{C^{\prime}}{K_{1}}\right) C_{s, p_{0}}+e^{a}\right]
$$

holds uniformly for $t \in[0, T]$, as the parameters in the right hand side are uniform in time. Choosing a small enough such that

$$
m_{0}+2 a\left[\left(1+\frac{C^{\prime}}{K_{1}}\right) C_{s, p_{0}}+e^{a}\right]<4 m_{0},
$$

or equivalently

$$
a<\min \left\{1, a_{0}, \frac{K_{1}}{2}, \frac{3 m_{0}}{2\left[\left(1+\frac{C^{\prime}}{K_{1}}\right) C_{s, p_{0}}+e^{a}\right]}\right\},
$$

where $K_{1}$ was defined in (12) and $C_{s, p_{0}}$ in (31), proves by definition of $T$ that $T=+\infty$ for any $n$. Passing to the limit $n \rightarrow+\infty$ concludes the proof.

In the general case $\beta \in(0,2]$, again as in the previous proof it follows that equation (30) has the extra positive term in the right hand side $K_{2} m_{s p}$. The corresponding equation to (32) is now

$$
\begin{aligned}
\frac{\mathrm{d}}{\mathrm{d} t} \sum_{p=p_{0}}^{n} m_{s p} \frac{a^{p}}{p !} \leq I_{s, \beta}^{n}(t, a)\left[4 \gamma_{s p_{0}} E_{s}^{n}(t, a)-\right. & \left.K_{1}\right] \\
& +K_{2} E_{s}^{n}(t, a)+\left(K_{1}+K_{2}\right) C_{s, p_{0}}
\end{aligned}
$$

and consequently, arguing as before we get

$$
\frac{\mathrm{d}}{\mathrm{d} t} \sum_{p=p_{0}}^{n} m_{s p} \frac{a^{p}}{p !} \leq\left(K_{2}-\frac{K_{1}}{2 a}\right) E_{s}^{n}(t, a)+K_{1}\left(\frac{m_{0}}{2 a}+e^{a}\right)+\left(K_{1}+K_{2}\right) C_{s, p_{0}} .
$$


In particular, making the additional restriction that $a<K_{1} /\left(4 K_{2}\right)$ we obtain the bound

$$
E_{s}^{n}(t, a) \leq 2 m_{0}+4 a\left[\left(1+\frac{K_{2}}{K_{1}}+\frac{C^{\prime}}{K_{1}}\right) C_{s, p_{0}}+e^{a}\right]
$$

uniformly for $t \in[0, T]$, where now $a$ is chosen so that

$$
a<\min \left\{1, a_{0}, \frac{K_{1}}{8 K_{2}}, \frac{m_{0}}{2\left[\left(1+\frac{K_{2}}{K_{1}}+\frac{C^{\prime}}{K_{1}}\right) C_{s, p_{0}}+e^{a}\right]}\right\},
$$

with $K_{1}$ and $K_{2}$ given in (11), with $p_{0}$ such that $\gamma_{s p_{0} / 2}<\left(32+2^{1-\beta}\right)^{-1}$. The proof is then completed as in the case $\beta \in(0,1]$ above.

\section{Appendix A. Some technical tools on moments}

We collect here two technical calculations from previous works.

Lemma 11 (Lemma 2 in 4). Assume that $p>1$, and let $k_{p}$ denote the integer part of $(p+1) / 2$. Then for all $x, y>0$ the following inequalities hold

$$
\sum_{k=1}^{k_{p}-1}\left(\begin{array}{l}
p \\
k
\end{array}\right)\left(x^{k} y^{p-k}+x^{p-k} y^{k}\right) \leq(x+y)^{p}-x^{p}-y^{p} \leq \sum_{k=1}^{k_{p}}\left(\begin{array}{l}
p \\
k
\end{array}\right)\left(x^{k} y^{p-k}+x^{p-k} y^{k}\right) .
$$

Lemma 12 (Lemma 2 in [6]). The energy-conserving solutions to the Boltzmann equation (11) on $[0,+\infty)$ with initial data $f^{0} \in L^{1}\left(1+|v|^{2}\right)$ satisfy

$$
\forall t \geq 0, \forall v \in \mathbb{R}^{d}, \quad \int_{\mathbb{R}^{d}} f\left(t, v_{*}\right)\left|v-v_{*}\right|^{s} \mathrm{~d} v_{*} \geq c_{s} \int_{\mathbb{R}^{d}} f^{0}\left(v_{*}\right)\left|v-v_{*}\right|^{s} \mathrm{~d} v_{*}
$$

for any $s \in(0,1]$ and some constant $c_{s}>0$ depending on $s$. This implies that

$$
\forall t \geq 0, \forall v \in \mathbb{R}^{d}, \quad \int_{\mathbb{R}^{d}} f\left(t, v_{*}\right)\left|v-v_{*}\right|^{s} \mathrm{~d} v_{*} \geq C_{f^{0}, s}\left(1+|v|^{s}\right)
$$

for any $s \in(0,1]$ and some constant $C_{f^{0}, s}>0$ depending on $s$ and the initial data $f^{0}$.

\section{REFERENCES}

[1] Alonso, R. J., ANd Lods, B. Free cooling and high-energy tails of granular gases with variable restitution coefficient. SIAM J. Math. Anal. 42, 6 (2010), 2499-2538.

[2] Bobylev, A. V. The theory of the nonlinear spatially uniform Boltzmann equation for Maxwell molecules. In Mathematical physics reviews, Soviet Sci. Rev. Sect. C Math. Phys. Rev. Harwood Academic Publishers, Chur, 1988, pp. 111-233.

[3] Bobylev, A. V. Moment inequalities for the Boltzmann equation and applications to spatially homogeneous problems. J. Stat. Phys. 88, 5 (1996), 1183-1214.

[4] Bobylev, A. V., Gamba, I. M., and Panferov, V. A. Moment inequalities and high-energy tails for Boltzmann equations with inelastic interactions. J. Stat. Phys. 116, 5 (2004), 16511682.

[5] Desvillettes, L. Some applications of the method of moments for the homogeneous Boltzmann and Kac equations. Arch. Rational Mech. Anal. 123, 4 (1993), 387-404.

[6] Gamba, I., Panferov, V., and Villani, C. Upper Maxwellian bounds for the spatially homogeneous Boltzmann equation. Arch. Rational Mech. Anal. 194, 1 (2009), 253-282.

[7] Ikenberry, E., And Truesdell, C. On the pressures and the flux of energy in a gas according to Maxwell's kinetic theory. I. J. Rational Mech. Anal. 5 (1956), 1-54.

[8] Lu, X., And Mounot, C. On measure solutions of the Boltzmann equation, part I: Moment production and stability estimates. J. Diff. Equations, 252 (2012), 3305-3363. 
[9] Mischler, S., And Mounot, C. Cooling process for inelastic Boltzmann equations for hard spheres. II. Self-similar solutions and tail behavior. J. Stat. Phys. 124, 2-4 (2006), 703-746.

[10] Mischler, S., And Wennberg, B. On the spatially homogeneous Boltzmann equation. Ann. Inst. H. Poincaré Anal. Non Linéaire 16, 4 (1999), 467-501.

[11] Моинот, C. Rate of convergence to equilibrium for the spatially homogeneous Boltzmann equation with hard potentials. Comm. Math. Phys. 261, 3 (2006), 629-672.

[12] Wennberg, B. Entropy dissipation and moment production for the Boltzmann equation. $J$. Stat. Phys. 86, 5 (1997), 1053-1066.

\author{
Ricardo Alonso \\ Institute for Pure And Applied Mathematics \\ (IPAM) UCLA - (CAAM) RiCE UNIVERSITY \\ 6100 Main Street - MS 134, Houston, TX 77005-1892, USA \\ EMAIL: rja2@rice.edu \\ José A. CAÑIzO \\ Centre for Mathematical Sciences \\ UNIVERSITY OF CAMBRIDGE \\ Cambridge CB3 0WA, UK \\ EMAIL: j.a.canizo@dpmms.cam.ac.uk \\ IRENE GAMBA \\ DePARTMENT OF MATHEMATICS \\ The University of Texas at Austin \\ TEXAS 78712, USA \\ E-MAIL: gamba@math.utexas.edu \\ Clément Mouhot \\ Centre for Mathematical Sciences \\ University OF CAMBridge \\ CAmbridge CB3 0WA, UK \\ EMAIL: C.Mouhot@dpmms. cam.ac.uk
}

